\title{
Genetic diversity of Echinococcus multilocularis in red foxes in Poland: the first report of a haplotype of probable Asian origin
}

\author{
Jacek Karamon, Krzysztof Stojecki, Małgorzata Samorek-Pieróg, Ewa Bilska-Zajac, Mirosław Rózycki, \\ Ewa Chmurzyńska, Jacek Sroka, Jolanta Zdybel and Tomasz Cencek
}

Department of Parasitology and Invasive Diseases, National Veterinary Research Institute, Puławy, Poland

\begin{abstract}
The aim of the present study was to estimate the genetic diversity of the cestode Echinococcus multilocularis Leuckart, 1863 in Poland based on sequence analysis of the mitochondrial genes of worms isolated from red foxes, Vulpes vulpes (Linnaeus). Overall, 83 adults of E. multilocularis from the same number of foxes in different parts of Poland were used for analysis. Sequences of the three mitochondrial genes, cytochrome $b(\mathrm{cob})$, NADH dehydrogenase subunit 2 (nad2) and cytochrome $c$ oxidase subunit 1 (cox)), were analysed. Seventy-four individual biological samples were successfully sequenced. Combined sequence analysis of these three genes exhibited fifteen Polish haplotypes (EmPL1-EmPL15). Most isolates $(\mathrm{n}=29 ; 39 \%)$ were classified to the EmPL1 haplotype, which occurred mainly in the east, north and centre of Poland. Haplotype EmPL4 $(n=14 ; 19 \%)$ and other haplotypes appeared predominantly in the south and west area. Fourteen haplotypes were grouped in the European clade. One Polish haplotype (EmPL9) (n=7, 10\%) was assigned to the Asian clade with haplotypes from Japan and Kazakhstan. This haplotype was found only in northeast Poland and this is the westernmost report of haplotype of E. multilocularis belonging to the Asian clade in Europe. The investigation demonstrated that populations of E. multilocularis in Poland (and probably also in eastern Europe) included not only different European haplotypes but also those of the Asian origin.
\end{abstract}

Keywords: echinococcosis, phylogenetics, Eastern Europe, Asian haplotype

This article contains supporting information (Figs. S1, S2) online at http://folia.paru.cas.cz/suppl/2017-64-007.pdf

Alveolar echinococcosis is a serious parasitic zoonosis caused by Echinococcus multilocularis Leuckart, 1863. This tapeworm is widely distributed in the northern hemisphere and the typical, final host of the parasite in Europe is the red fox, Vulpes vulpes (Linnaeus) (Oksanen et al. 2016). Humans play the role of aberrant, intermediate host in the parasite life cycle. In a recent study in Poland, E. multilocularis was found in relatively high percentages in red foxes - in some provinces the prevalence reached up to approximately 50\% (Karamon et al. 2014, 2015). Moreover, more than one hundred cases of human alveolar echinococcosis (AE) were described (Nahorski et al. 2013) - a relatively small number in comparison to the high prevalence of the parasite in red foxes. However, it must be stressed that most human AE records in Poland were detected sporadically and not in a planned monitoring study.

In the last several years, increasing research efforts have focused on the genetic diversity of E. multilocularis. So far, there has been no convincing data about the relevance of the parasite's different genetic groups in terms of public health (human infectivity, pathogenesis or drug efficacy). However, many studies conducted on E. multilocularis in- dicated a genetic variability associated with geographical distribution, which is connected, inter alia, with the specific movement routes of the parasite hosts. There are some different ways to analyse this phenomenon. For example, the genetic diversity at the continental scale and in single countries was estimated using a tandem repeat multi-loci microsatellite analysis (EmsB) (Knapp et al. 2008, Casulli et al. 2010, Umhang et al. 2014). EmsB investigation helped draw the theory of 'mainland-island' distribution of genotypes of E. multilocularis in Europe, which explained the imbalance of genetic diversity between ancestral and peripheral regions (Knapp et al. 2009).

Sequence analysis of the selected genes also provided much information about the genetic diversity of this parasite (Bowles et al. 1992, Haag et al. 1997, Gesy et al. 2014). Nakao et al. (2009) focused on three mitochondrial genes, cytochrome $c$ oxidase subunit 1 ( $\operatorname{cox} 1)$, NADH dehydrogenase subunit 2 (nad2) and cytochrome $b(c o b)$, identified 18 haplotypes grouped in four basal clades: Europe, Asia, North America and China (Inner Mongolia). Continuation of such an approach, which uses the same set of genes or a selected group, was carried out among others in Estonia, 


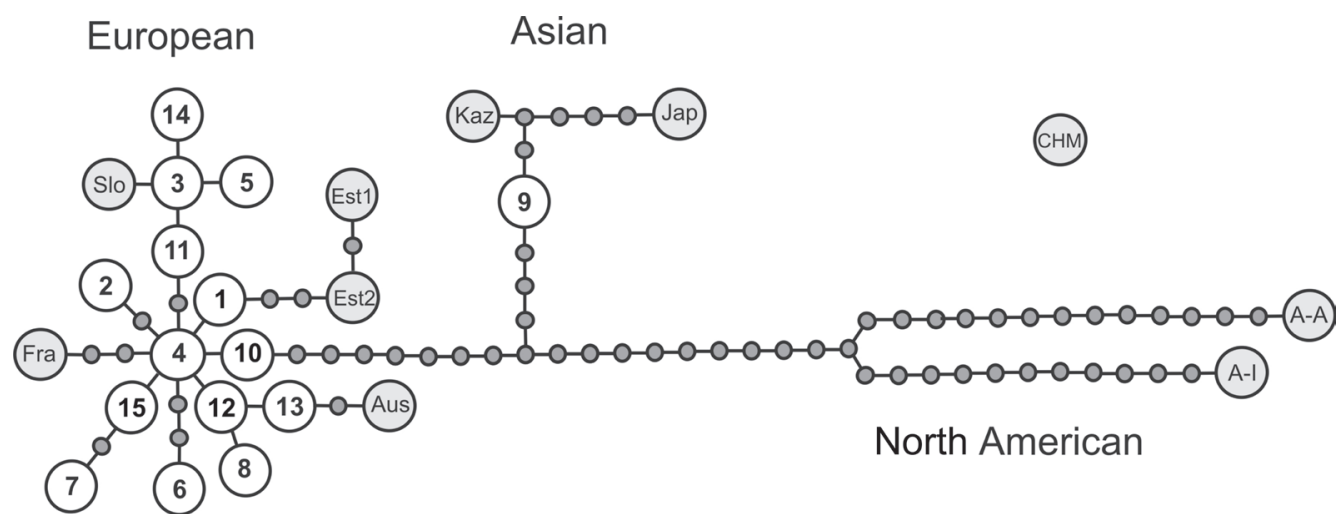

Fig. 1. The haplotype network of Echinococcus multilocularis Leuckart, 1863 constructed from a data set of cob, nad2 and coxl genes based on statistical parsimony. 1-15 - Polish haplotypes EmPL1-EmPL15; A-A - USA, Alaska (St. Lawrence Island); A-I - USA, Indiana; Aus - Austria; CHM - China (Inner Mongolia); Est1, Est2 - Estonia; Fra - France; Jap - Japan (Hokkaido); Kaz - Kazakhstan; Slo - Slovakia.

Canada and in a wide territory of Russia (Jenkins et al. 2012, Konyaev et al. 2013, Laurimaa et al. 2015).

On the one hand, the genetic diversity in Europe is explained by the mainland-island migration from the historical centre in Switzerland and Swabian Jura to peripheral areas, also eastward (Knapp et al. 2009). On the other hand, the analysis conducted in Asia and Europe (Nakao et al. 2009, Konyaev et al. 2013) suggests the spread of Asian genotypes from eastern Asia to the west (towards Europe). Especially noteworthy are the recent reports of isolates belonging to the Asian clade in the European part of Russia (southeast of Moscow, Ryazan Oblast) (Konyaev et al. 2013). It seems that thorough analysis of sufficiently numerous populations of E. multilocularis in eastern Europe can provide an answer regarding how far west the distribution of Asian populations reaches. Therefore, Poland with its geographical location (central-east Europe) is an interesting area for such investigations.

The aim of this study was to estimate the genetic diversity of E. multilocularis in Poland based on sequence analysis of the three mitochondrial genes (cob, nad2, cox 1$)$ of worms isolated from red foxes.

\section{MATERIALS AND METHODS}

Samples of Echinococcus multilocularis. Adults of E. multilocularis were obtained from red foxes from different parts of Poland. The tapeworms were collected during a national monitoring study carried out from 2009 to 2014 (Karamon et al. 2014, 2015). Tapeworms were isolated from the intestines using the sedimentation and counting technique (Hofer et al. 2000, OIE 2008) and preserved in $70 \%$ ethanol. Next, before DNA extraction, single tapeworms were washed in physiological saline in a Petri dish. Overall, 83 adults of E. multilocularis (one worm from each red fox) were prepared and used for analysis.

PCR and sequencing. DNA was extracted from each parasite and purified using a QIAamp DNA Stool Mini Kit (Qiagen, Hilden, Germany), according to the manufacturer's protocol. Three mitochondrial genes: $c o b$, nad2 and coxl, were amplified by PCR according to Nakao et al. (2009). Samples for sequencing were purified on Sephadex G-50 columns. Sequencing was performed using a BigDye ${ }^{\mathrm{TM}}$ Terminator v3.1 Cycle Sequencing kit
(Applied Biosystems, Foster City, USA) on an ABI3730xl Genetic Analyser (Applied Biosystems).

Data analysis (phylogenetic and haplotype analysis). For single locus and multilocus analysis, phylogeny has been reconstructed by the following methodology. Previously trimmed sequences were combined and aligned with ClustalW 2.1 software (Larkin et al. 2007) using the following parameters: Gap Opening Penalty -10 and Gap Extension Penalty - 0.2. Phylogenetic analysis was performed using RAxML version 8.0.19 (Stamatakis 2014). To obtain reliable multilocus typing, partitioned analysis was performed. For the guide tree and bootstrap, the GTRGAMMA model was selected, whereas partitioned analysis used the GTRCAT model. One thousand nonparametric bootstrap inferences were made. Haplotype inference of genetic variation was performed with the R-CRAN utilising package 'haplotypes' (Caner 2015, R-Core-Team 2015), with the parsimony analysis connection limit set on $95 \%$.

Nucleotide sequence data reported in this paper are available in the GenBank ${ }^{\mathrm{TM}}$ database under the accession numbers: KY205662-KY205706. To estimate the phylogenetic position of the Polish isolates, homologous mitochondrial DNA sequences described earlier (Xiao et al. 2005, Nakao et al. 2009, Jenkins et al. 2012, Konyaev et al. 2013, Laurimaa et al. 2015) were retrieved from GenBank and used in analyses.

\section{RESULTS}

Based on analysis of the combined sequences of the three genes (cob, nad2 and cox 1), fifteen haplotypes (EmPL1-EmPL15) of E. multilocularis were detected among the 74 isolates originating from Poland (Figs. 1-3).

Analysis showed that the most Polish isolates $(\mathrm{n}=29$; $39 \%$ ) were classified to the haplotype EmPL1. The next in numbers are haplotype EmPL4 $(\mathrm{n}=14 ; 19 \%)$ and EmPL9 $(\mathrm{n}=7 ; 10 \%)$. One Polish haplotype (EmPL9) was localised in the Asian clade with Japanese and Kazakhstan haplotypes. The remaining 14 haplotypes were grouped in the European clade. For example: EmPL3, 5, 11 and 14 were closely related to the Slovak haplotype, EmPL8, 12 and 13 to the Austrian haplotype, and EmPL1 to the Estonian haplotype. 


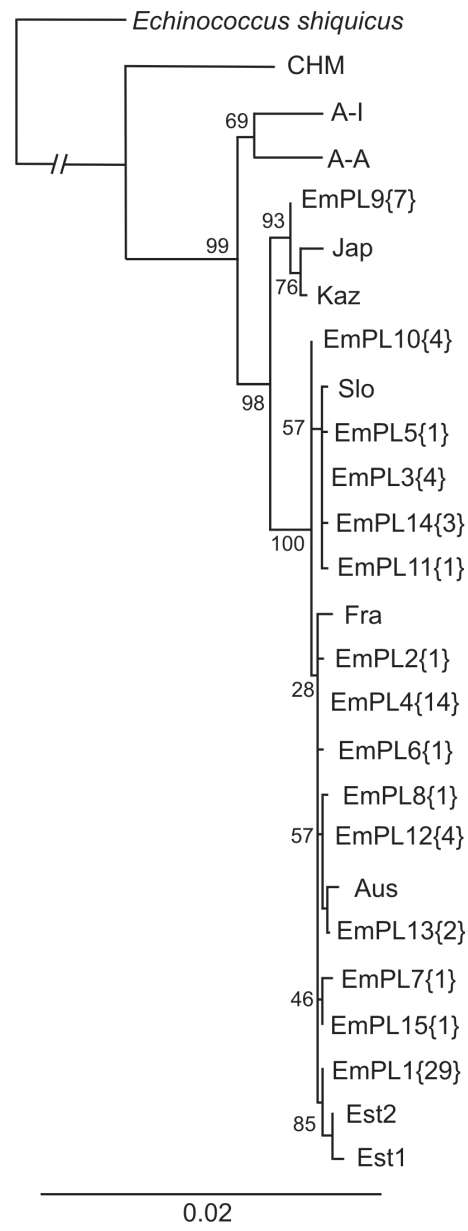

Fig. 2. A phylogenetic tree of Echinococcus multilocularis Leuckart, 1863 constructed from the data set of $c o b$, nad 2 and coxl genes. A-A - USA; Alaska (St. Lawrence Island); A-I - USA, Indiana; Aus - Austria; CHM - China (Inner Mongolia); EmPL1EmPL15 - Polish haplotypes (numbers of Polish isolates in each haplotype are shown in brace brackets \{\} ); Est1, Est2 - Estonia; Fra - France; Jap - Japan (Hokkaido); Kaz - Kazakhstan; Slo-Slovakia. Values on the tree nodes are bootstrap proportions (\%).

The map (Fig. 4) shows that the distinct occurrence of individual haplotypes is related with geographical locations. The Asian haplotype (EmPL9) was found only in northeast Poland. The dominant haplotype (EmPL1) occurred in a majority of the Polish territory - east, north and centre; however, single EmPL1 isolates were found in the south and west only. Other haplotypes (also the second most numerous - EmPL4) appeared predominantly only in the south and west area.

During comparison of individual genes, the number of haplotypes was lower than in the combined sequences. There were seven haplotypes in the coxl gene (82 isolates analysed) (Fig. 3), nine in cob (79 isolates analysed) and four in nad2 (79 isolates analysed) (Figs. S1, S2).

All seven isolates in the combined analysis of the three genes that were identified as the Asian haplotype (EmPL9) were also classified in Asian clade in a separate analysis of the individual genes cox $1, c o b$ and nad2. In cox 1 and nad2 analysis, one additional isolate (which was not used in combined analysis because of a poor quality cob sequence)

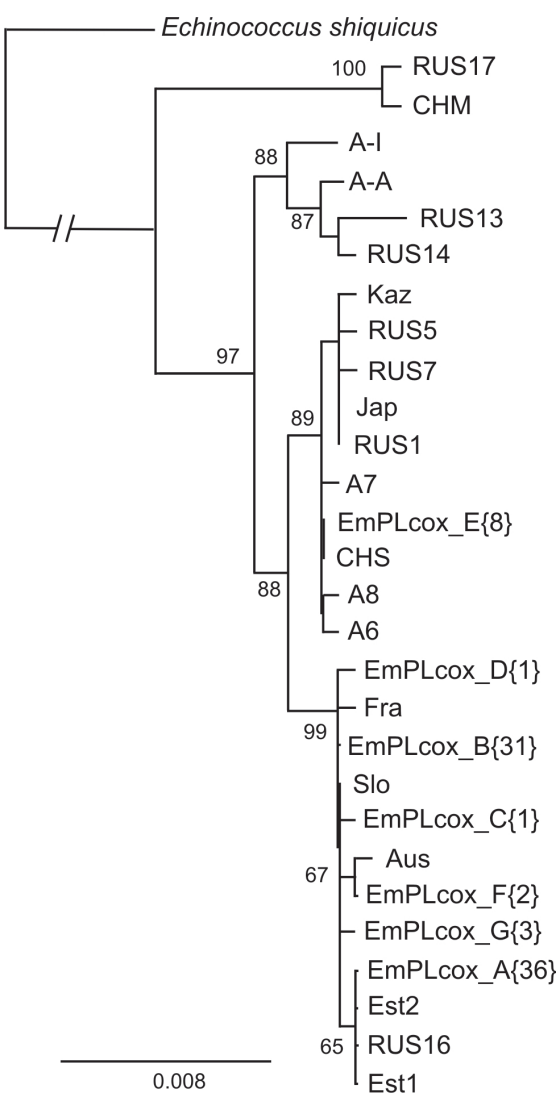

Fig. 3. A phylogenetic tree of Echinococcus multilocularis Leuckart, 1863 based on the coxl gene. A-A - USA, Alaska (St. Lawrence Island); A-I - USA, Indiana; Aus - Austria; CHM - China (Inner Mongolia); CHS, A6-A8 - China (Sichuan); EmPLcoxA-EmPLcox_G - Polish haplotypes (numbers of Polish isolates in each haplotype are shown in brace brackets \{\}$)$; Est1, Est2 - Estonia; Fra - France; Jap - Japan (Hokkaido), Kaz - Kazakhstan; RUS1, RUS5, RUS7, RUS 13, RUS14, RUS16, RUS17 - Russia; Slo-Slovakia. Values on the tree nodes are bootstrap proportions $(\%)$.

was classified to the Asian clade. In approximately $70 \%$ of all isolates in both separated analyses ( $c o b$ and nad2) were located in one dominant haplotype. These isolates were generally grouped to the most numerous haplotypes of the multilocus analysis: EmPL1 and EmPL4. Results of the separated coxl analysis were closer to the common analysis of the three genes. For example, a dominant cox 1 haplotype (EmPLcox_A) was constructed from the same isolates as EmPL1 (and some additional isolates which did not participate in the combined analysis) (Fig. 3). The main clades in phylogenetic trees were supported with high bootstrap values. However, some external branching nodes were not so highly supported with relatively low bootstrap.

\section{DISCUSSION}

The present study describes the genetic diversity of Echinococcus multilocularis in Poland. Most of the described isolates belonged to the European clade; however, one haplotype (EmPL9) was classified to the Asian group. This haplotype is most similar to haplotypes identified in 


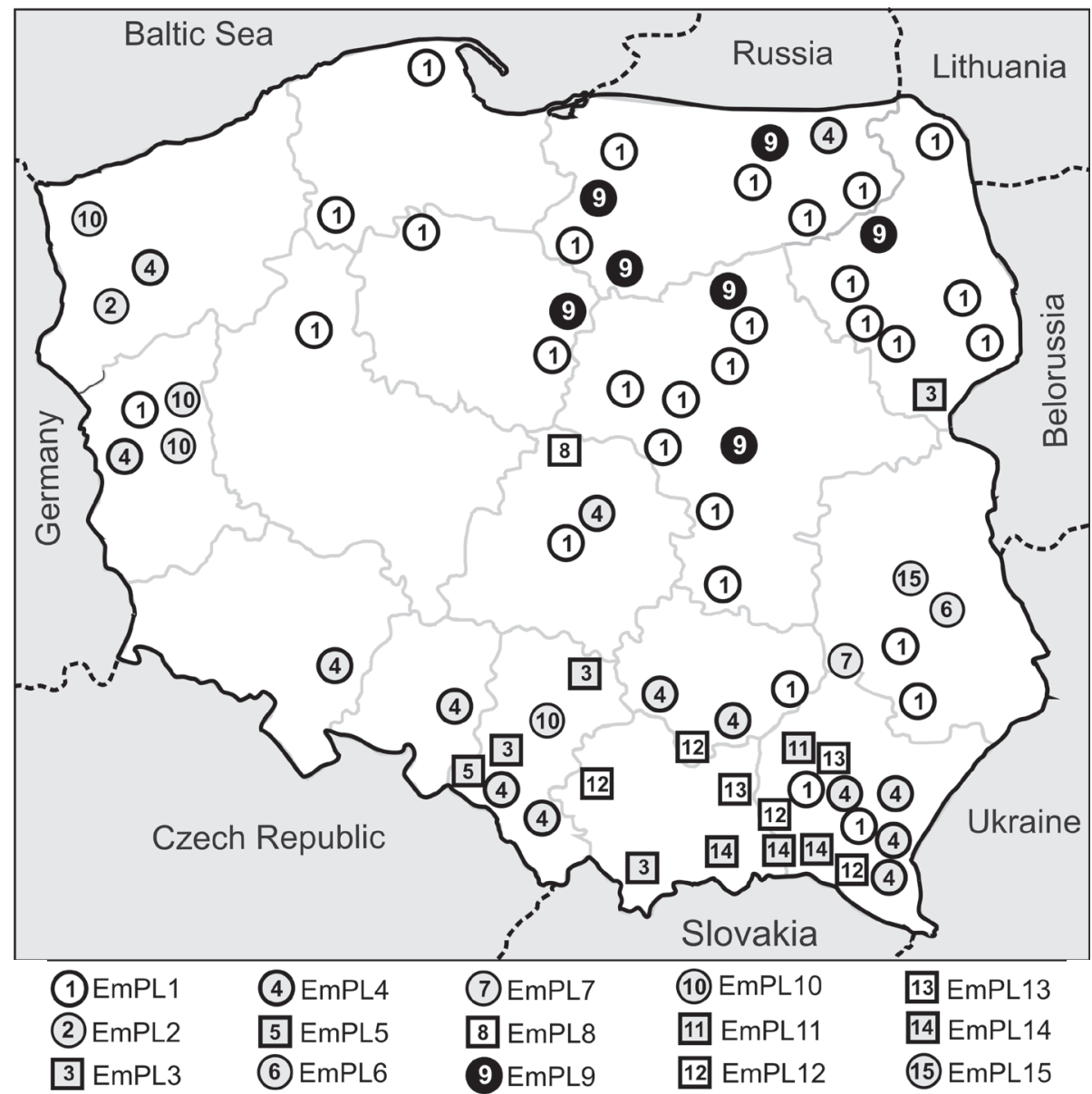

Fig. 4. Geographic distribution of Echinococcus multilocularis Leuckart, 1863 haplotypes in Poland. Numbers on the map (1-15) correspond to the respective haplotypes (EmPL1-EmPL15).

samples from Kazakhstan and Japan. Moreover, isolates belonging to this haplotype, in a separate analysis of cox 1 gene, were also closely related to samples from China (Sichuan) (Nakao et al. 2009) and numerous isolates from Russia (Konyaev et al. 2013). The European haplotype from this clade (EmRUS1) was already identified earlier, but it was approximately $1,400 \mathrm{~km}$ to the east of Poland (Ryazan Oblast, Russia) (Konyaev et al. 2013). Haplotype EmPL9 occurred only in the northeast part of Poland and, till now, was the westernmost location of any member of the Asian cluster of haplotypes in Europe. The results of the present study (distribution in a limited northeast area) and no other available data concerning these haplotypes in central and western Europe could suggest that this is a western borderline of distribution of haplotypes coming from Asia.

The dominant Polish haplotype (EmPL1) is observed in the vast majority of the area in the north, northeast and centre of Poland and shares this territory mainly with the Asian (EmPL9) haplotype. EmPL1 is very similar to the Estonian haplotypes (Laurimaa et al. 2015) and is not closely related to most of the European isolates described previously (Nakao et al. 2009). Comparison of the cox 1 sequences also indicates that there is high similarity of EmPLcoxA (containing all samples belonging to EmPL1) to the RUS16 haplotype detected in the Moscow zoo in a Senegal bushbaby (Galago senegalensis Geoffroy) (Konyaev et al. 2013). The authors suggested that the most likely source of infection for this monkey was the mulch used in the cage, which occasionally came from the Baltic countries (Latvia and Estonia). It may explain the similarity with the Estonian, and thus the EmPL1, isolates.

In the south, there are haplotypes closely related to the isolates found earlier in southern Europe (Austria and Slovakia) and in France (Nakao et al. 2009). In addition, the second most numerous Polish haplotype (EmPL4) is located mainly in the south and west. The geographical location of EmPL4 (and less numerous EmPL10) can confirm the results of the genetic analysis (Figs. 1,2) suggesting that they are a sort of transition haplotypes between the dominant haplotype in Poland - EmPL1 (characteristic for the northeastern part of the country, as well as the northeastern Europe - Estonia) and the haplotypes of southern and western Europe.

There are assumptions that the separation of the European and Asian clades of E. multilocularis occurred probably 60,000-37,000 years ago during the Last Glacial Maximum. In this period, red foxes and many other animal species withdrew to places outside of the glacial ice zone (to glacial refugia). In Europe, refugia were located in the southern part of the continent. Isolation of red fox populations occurred approximately 10,000 years (Sommer 
and Benecke 2005). After warming, the animals emerged from these places and re-colonised the continent, probably bringing with them the genetic variations of their parasites. This genetic diversity was shown in the European study analysing mitochondrial sequences (Nakao et al. 2009), and also with the use of microsatellite analysis (Knapp et al. 2009).

On the other hand, there is a hypothesis that the Asian haplotypes of E. multilocularis were spread in Asia through the foxes re-colonising the continent, among others, from the Bering Land formed by glaciation ('Beringia'), which acted as a refugium for East Siberia (Nakao et al. 2009). Asian haplotypes detected in Kazakhstan, Japan, China and Russia were found also on St. Lawrence Island in the Bering Sea (Nakao et al. 2009, Konyaev et al. 2013). Based on this hypothesis, it can be assumed that the Asian haplotype found in Poland (EmPL9) reached the eastern regions of central Europe together with the red foxes migrating from Asia to the west. Moreover, it should be stressed that the European clade is represented by fourteen haplotypes, whereas the Asian clade is represented only by one. This argues for a recent introduction of the Asian population to this area.

The investigation indicated that the genetic diversity of E. multilocularis in Poland (and probably also in eastern Europe) was co-created by penetration of Asian haplotypes. Therefore, on the one hand, there are haplotypes from western and southern Europe, which are a continuation of the historical migration from the endemic European core area (explained by hypothesis mainland-island system - Knapp et al. 2009). On the other hand, genetic diversity in this area is created also by the parasite populations coming from the East (Asia). Of course, to have more knowledge about the distribution and migration of populations of E. multilocularis in the wildlife, further studies with more samples from Europe, especially the eastern regions, are required.

Acknowledgements. The investigation was supported by the Polish Ministry of Agriculture and Rural Development (in the frame of the Multiannual Programme 'Protection of Animal and Public Health').

\section{REFERENCES}

Bowles J., Blair D., McManus D.P. 1992: Genetic-variants within the genus Echinococcus identified by mitochondrial-DNA sequencing. Mol. Biochem. Parasitol. 54: 165-174.

CANer A. 2015: Haplotypes: Haplotype inference and statistical analysis of genetic variation. $\mathrm{R}$ package version 1.0. http:// CRAN.R-project.org/package $=$ haplotypes

Casulli A., Szell Z., Pozio E., Sreter T. 2010: Spatial distribution and genetic diversity of Echinococcus multilocularis in Hungary. Vet. Parasitol. 174: 241-246.

Gesy K.M., Schurer J.M., Massolo A., Liccioli S., Elkin B.T., Alisauskas R., Jenkins E.J. 2014: Unexpected diversity of the cestode Echinococcus multilocularis in wildlife in Canada. J. Parasitol. Par. Wildl. 3: 81-87.

Haag K.L., Zaha A., Araujo A.M., Gottstein B. 1997: Reduced genetic variability within coding and non-coding regions of the Echinococcus multilocularis genome. Parasitology 115: 521-529.

Hofer S., Gloor S., Muller U., Mathis A., Hegglin D., Deplazes P. 2000: High prevalence of Echinococcus multilocularis in urban red foxes (Vulpes vulpes) and voles (Arvicola terrestris) in the city of Zurich, Switzerland. Parasitology 120: $135-142$.

Jenkins E.J., Peregrine A.S., Hill J.E., Somers C., Gesy K., Barnes B., Gottstein B., Polley L. 2012: Detection of European strain of Echinococcus multilocularis in North America. Emerg. Infect. Dis. 18: 1010-1012.

Karamon J., Kochanowski M., Dabrowska J., Sroka J., Różycki M., Bilska-Zając E., Cencek T. 2015: Dynamics of Echinococcus multilocularis infection in red fox populations with high and low prevalence of this parasite in Poland (20072014). B. Vet. I. Pulawy 59: 213-217.

Karamon J., Kochanowski M., Sroka J., Cencek T., RóżyCKi M., ChmurzyńsKa E., BilsKa-ZająC E. 2014: The prevalence of Echinococcus multilocularis in red foxes in Poland current results (2009-2013). Parasitol. Res. 113: 317-322.

Knapp J., Bart J.-M., Giraudoux P., Glowatzki M.-L., Breyer I., Raoul F., Deplazes P., Duscher G., Martinek K., Dubinsky P., Guislain M.-H., Cliquet F., Romig T., MalCzewski A., Gottstein B., Piarroux R. 2009: Genetic diversity of the cestode Echinococcus multilocularis in red foxes at a continental scale in Europe. PloS Negl. Trop. Dis. 3: e452.
Knapp J., Guislain M.H., Bart J.M., Raoul F., Gottstein B., Giraudoux P., Piarroux R. 2008: Genetic diversity of Echinococcus multilocularis on a local scale. Infect. Genet. Evol. 8: 367-373.

Konyaev S.V., Yanagida T., Nakao M., Ingovatova G.M., Shoykhet Y.N., Bondarev A.Y., Odnokurtsev V.A., Loskutova K.S., Lukmanova G.I., Dokuchaev N.E., SpiriDONOv S., Alshinecky M.V., Sivkova T.N., Andreyanov O.N., Abramov S.A., Krivopalov A.V., Karpenko S.V., Lopatina N.V., Dupal T.A., Sako Y., Ito A. 2013: Genetic diversity of Echinococcus spp. in Russia. Parasitology 140: $1637-1647$.

Larkin M.A., Blackshields G., Brown N.P., Chenna R., McGettigan P.A., McWilliam H., Valentin F., Wallace I.M., Wilm A., Lopez R., Thompson J.D., Gibson T.J., HigGINS D.G. 2007: Clustal W and Clustal X version 2.0. Bioinformatics 23: 2947-2948.

Laurimaa L., Sueld K., Moks E., Valdmann H., Umhang G., Knapp J., SaArma U. 2015: First report of the zoonotic tapeworm Echinococcus multilocularis in raccoon dogs in Estonia, and comparisons with other countries in Europe. Vet. Parasitol. 212: 200-205.

Nahorski W.L., Knap J.P., Pawlowski Z.S., Krawczyk M., Polanski J., Stefaniak J., Patkowski W., Szostakowska B., Pietkiewicz H., Grzeszczuk A., Felczak-Korzybska I., Golab E., Wnukowska N., Paul M., Kacprzak E., Sokolewicz-Bobrowska E., Niscigorska-Olsen J., Czyrznikowska A., Chomicz L., Cielecka D., Myjak P. 2013: Human alveolar echinococcosis in Poland: 1990-2011. PloS. Negl. Trop. Dis. 7: e1986.

NaKao M., XiaO N., OKamoto M., Yanagida T., Sako Y., Ito A. 2009: Geographic pattern of genetic variation in the fox tapeworm Echinococcus multilocularis. Parasitol. Int. 58: 384-389.

OIE 2008: Echinococcosis/hydatidosis (infection with Echinococcus granulosus and E. multilocularis). In: Manual of Diagnostic Tests and Vaccines for Terrestrial Animals. Office International des Epizooties, Paris, pp. 1-15

Oksanen A., Siles-Lucas M., Karamon J., Possenti A., Conraths F.J., Romig T., Wysocki P., Mannocci A., Mipatrini D., La Torre G., Boufana B., Casulli A. 2016: The geographical distribution and prevalence of Echinococcus multiloc- 
ularis in animals in the European Union and adjacent countries: a systematic review and meta-analysis. Par. Vect. 9: 1-23.

R-Core-Team 2015: R: A language and environment for statistical computing. R Foundation for Statistical Computing, Vienna, https://www.R-project.org/.

Sommer R., Benecke N. 2005: Late-Pleistocene and early Holocene history of the canid fauna of Europe (Canidae). Mamm. Biol. 70: 227-241.

Stamatakis A. 2014: RAxML version 8: a tool for phylogenetic analysis and post-analysis of large phylogenies. Bioinformatics 30: $1312-1313$.
Umhang G., Knapp J., Hormaz V., Raoul F., Boue F. 2014: Using the genetics of Echinococcus multilocularis to trace the history of expansion from an endemic area. Inf. Gen. Evol. 22: $142-149$.

Xiao N., Qiu J.M., Nakao M., Li T.Y., Yang W., Chen X.W., Schantz P.M., Craig P.S., Ito A. 2005: Echinococcus shiquicus $\mathrm{n}$. sp., a taeniid cestode from Tibetan fox and plateau pika in China. Int. J. Parasitol. 35: 693-701.

Cite this article as: Karamon J., Stojecki K., Samorek-Pieróg M., Bilska-Zajac E., Rózycki M., Chmurzyńska E., Sroka J., Zdybel J., Cencek T. 2017: Genetic diversity of Echinococcus multilocularis in red foxes in Poland: the first report of a haplotype of probable Asian origin. Folia Parasitol. 64: 007. 